\title{
Hyperelliptic modular curves $X_{0}^{*}(N)$
}

by

\author{
Yuji HaSEGawa (Tokyo)
}

1. Introduction. Let $N$ be a positive integer, and let

$$
\Gamma_{0}(N)=\left\{\left(\begin{array}{ll}
a & b \\
c & d
\end{array}\right) \in \mathrm{SL}_{2}(\mathbb{Z}) \mid c \equiv 0 \bmod N\right\} .
$$

For each positive divisor $N^{\prime}$ of $N$ with $\left(N^{\prime}, N / N^{\prime}\right)=1$ (we write $N^{\prime} \| N$ ), $W_{N^{\prime}}=W_{N^{\prime}}^{(N)}$ denotes the corresponding Atkin-Lehner involution defined for $\Gamma_{0}(N)$. ( $W_{1}$ is the identity operator.) Then we define the modular group $\Gamma_{0}^{*}(N)$ to be the group generated by $\Gamma_{0}(N)$ and $\left\{W_{N^{\prime}}\right\}_{N^{\prime} \| N}$ :

$$
\Gamma_{0}^{*}(N)=\left\langle\Gamma_{0}(N) \cup\left\{W_{N^{\prime}}\right\}_{N^{\prime} \| N}\right\rangle .
$$

It is well known (see [1]) that $\Gamma_{0}^{*}(N)$ normalizes $\Gamma_{0}(N)$ and the factor group $W(N):=\Gamma_{0}^{*}(N) / \Gamma_{0}(N)$ is abelian of type $(2, \ldots, 2)$ with order $2^{\omega(N)}$, where $\omega(N)$ denotes the number of the distinct prime divisors of $N$.

Let $X_{0}^{*}(N)$ be the modular curve which corresponds to $\Gamma_{0}^{*}(N)$, namely,

$$
X_{0}^{*}(N):=X_{0}(N) / W(N)=X_{0}(N) /\left\langle\left\{W_{N^{\prime}}\right\}_{N^{\prime} \| N}\right\rangle .
$$

In [7], we proved

Theorem A. Assume that $N$ is square-free. Then $X_{0}^{*}(N)$ is hyperelliptic if and only if $X_{0}^{*}(N)$ is of genus two.

In [7], we were reduced to 56 cases (21 square-free cases and 35 nonsquare-free cases). The above theorem, conjectured by Kluit [9], is the result for square-free cases.

The purpose of this article is to determine all hyperelliptic curves of type $X_{0}^{*}(N)$ with genus $\geq 3$, i.e., to check the hyperellipticity of $X_{0}^{*}(N)$ for the 35 values of $N$ listed in Table 1 . Our result is formulated in Theorem B below.

1991 Mathematics Subject Classification: Primary 11F11; Secondary 11F06, 11G30, 14H45, 14G05, 14H25. 
Table 1

\begin{tabular}{c|cccccccccc}
\hline Genus & \multicolumn{110}{|c}{$N$} \\
\hline 3 & 136 & 144 & 152 & 162 & 164 & 171 & 175 & 196 & 207 & 234 \\
& 240 & 252 & 270 & 294 & 312 & 315 & 348 & 420 & 476 & \\
4 & 160 & 176 & 264 & 280 & 300 & 306 & 342 & & & \\
5 & 216 & 279 & 396 & 630 & & & & & & \\
6 & 336 & & & & & & & & & \\
7 & 360 & 450 & & & & & & & & \\
10 & 840 & & & & & & & & & \\
19 & 1680 & & & & & & & & & \\
\hline
\end{tabular}

Theorem B. There are sixty-four values of $N$ for which $X_{0}^{*}(N)$ is hyperelliptic. Of these, there are only seven values of $N$ for which $X_{0}^{*}(N)$ is hyperelliptic with genus $g \geq 3$, namely, $N=136,171,207,252,315$ for $g=3, N=176$ for $g=4$, and $N=279$ for $g=5$.

Remark 1. The 57 values of $N$ for which $X_{0}^{*}(N)$ is of genus two are as follows:

$$
\begin{aligned}
& 67, \quad 73,85,88, \quad 93,103,104,106,107,112 \text {, } \\
& 115,116,117,121,122,125,129,133,134,135 \text {, } \\
& 146,147,153,154,158,161,165,166,167,168 \text {, } \\
& 170,177,180,184,186,191,198,204,205,206 \text {, } \\
& 209,213,215,221,230,255,266,276,284,285 \\
& 286,287,299,330,357,380,390 .
\end{aligned}
$$

Their defining equations are given in [5] (see also [11]).

Notation. $\mathbb{Z}, \mathbb{Q}, \mathbb{C}$ denote respectively the ring of rational integers, the field of rational numbers and the field of complex numbers. $\mathbb{F}_{p^{\nu}}$ denotes the finite field with $p^{\nu}$ elements. $\mathbb{P}^{n}$ is the $n$-dimensional projective space. We denote by $\tau$ an element of the complex upper half plane, and we put $q=\exp (2 \pi i \tau)$.

2. Modular involutions on $X_{0}^{*}(N)$ (I). In this section, we treat the case with $8 \mid N$ or $9 \| N$. As we shall show, $X_{0}^{*}(N)$ has an involution which comes from a matrix when $8 \mid N$ or $9 \| N$. We can use this involution to determine the hyperellipticity of $X_{0}^{*}(N)$ for some cases. One may refer to [10], [1] for the structure of the normalizer of $\Gamma_{0}(N)$ in $\mathrm{GL}_{2}^{+}(\mathbb{Q})$. But he should be careful to use Theorem 8 of [1], since some errors are included there.

Put $S_{\mu}=\left(\begin{array}{ll}\mu & 1 \\ 0 & \mu\end{array}\right)$. Then $S_{2}$ is in the normalizer of $\Gamma_{0}(N)$ when $N$ is divisible by 4 , and $S_{3}$ is in the normalizer of $\Gamma_{0}(N)$ when $N$ is divisible by 9 .

Proposition 1. (i) Let $2^{\nu} \| N$ with $\nu \geq 3$. Then $V_{2}=S_{2} W_{2^{\nu}} S_{2}$ normalizes $\Gamma_{0}^{*}(N)$ and $\Gamma_{0}(N)$. Further, $V_{2}^{2} \in \Gamma_{0}(N)$.

(ii) Let $9 \| N$. Then $V_{3}=S_{3} W_{9} S_{3}^{2}$ normalizes $\Gamma_{0}^{*}(N)$ and $\Gamma_{0}(N)$. Further, $V_{3}^{2} \in \Gamma_{0}(N)$. 
Proof. This follows from a direct calculation.

Corollary. Suppose $8 \mid N$ (resp. $9 \| N)$. Then $V_{2}$ (resp. $\left.V_{3}\right)$ defines an involution on $X_{0}(N)$ and on $X_{0}^{*}(N)$.

Let $S_{2}(N), S_{2}^{0}(N)$ and $S_{2}^{*}(N)$ be respectively the space of cuspforms of weight 2 on $\Gamma_{0}(N)$, the space spanned by newforms of weight 2 on $\Gamma_{0}(N)$, and the space of cuspforms of weight 2 on $\Gamma_{0}^{*}(N)$. Put $V=V_{2}$ or $V_{3}$ (or $V_{2} V_{3}=V_{3} V_{2}$ if $\left.N=144=2^{4} 3^{2}\right)$. To calculate the genus $\bar{g}=\bar{g}(N ; V)$ of $X_{0}^{*}(N) /\langle V\rangle$, it suffices to determine the dimension of the subspace

$$
S_{2}^{*}(N)^{V}=\left\{f \in S_{2}^{*}(N)|f| V=f\right\}
$$

of $S_{2}^{*}(N)$.

Lemma 1. Let $M$ be a positive integer. Let $M^{\prime}$ be a positive divisor of $M$ and let $d$ be a positive divisor of $M / M^{\prime}$. For a prime divisor $p$ of $M$, define integers $\alpha, \beta$ and $\gamma$ by

$$
p^{\alpha}\left\|M, \quad p^{\alpha-\beta}\right\| M^{\prime}, \quad p^{\gamma} \| d .
$$

(i) Let $f(\tau) \in S_{2}\left(M^{\prime}\right)$. Then

$$
f(d \tau) \mid W_{p^{\alpha}}^{(M)}=p^{\beta-2 \gamma}\left(f \mid W_{p^{\alpha-\beta}}^{\left(M^{\prime}\right)}\right)\left(d^{\prime} \tau\right),
$$

where $d^{\prime}=p^{\beta-2 \gamma} d$.

(ii) If $f \in S_{2}^{0}\left(M^{\prime}\right)$ is a newform on $\Gamma_{0}\left(M^{\prime}\right)$, then $f$ is also an eigenform for all $W_{m}^{\left(M^{\prime}\right)}$ with $m \| M^{\prime}$. In particular, if $f \mid W_{p^{\alpha-\beta}}^{\left(M^{\prime}\right)}=\lambda_{p}^{\prime} f(= \pm f)$ and if $\beta \neq 2 \gamma($ resp. $\beta=2 \gamma)$, then

$$
f(d \tau) \pm p^{\beta-2 \gamma} \lambda_{p}^{\prime} f\left(d^{\prime} \tau\right) \quad(\text { resp. } f(d \tau))
$$

becomes an eigenform for $W_{p^{\alpha}}^{(M)}$ with eigenvalue equal to \pm 1 (resp. $\left.\lambda_{p}^{\prime}\right)$.

Proof. See [1].

For simplicity, we will sometimes write $f^{(d)}(\tau)=f(d \tau)$ in the following.

Proposition 2. Let $N$ be a positive integer such that $8 \mid N$. Let $N^{\prime}$ be a positive divisor of $N$ and let $d$ be a positive divisor of $N / N^{\prime}$. Define integers $\alpha, \beta$ and $\gamma$ by

$$
2^{\alpha}\left\|N, \quad 2^{\alpha-\beta}\right\| N^{\prime}, \quad 2^{\gamma} \| d
$$

so that $N=2^{\alpha} M$ and $N^{\prime}=2^{\alpha-\beta} M^{\prime}$ for some positive odd integers $M, M^{\prime}$ with $M^{\prime} \mid M$. Let $f=\sum a_{n} q^{n}$ be a newform on $\Gamma_{0}\left(N^{\prime}\right)$ such that $f \mid W_{2^{\alpha-\beta}}^{\left(N^{\prime}\right)}=$ $\lambda f$, and put

$$
g^{(d)}=f^{(d)}+f^{(d)} \mid W_{2^{\alpha-\beta}}^{\left(N^{\prime}\right)}=f^{(d)}+2^{\beta-2 \gamma} \lambda f^{\left(d^{\prime}\right)}
$$

with $d^{\prime}=2^{\beta-2 \gamma} d$. 
(i) If $\alpha-\beta \geq 2$, then

$$
\begin{cases}g^{(d)} \mid V_{2}=-g^{(d)} & \text { if } \beta>\gamma=0 \\ g^{(d)} \mid V_{2}=+g^{(d)} & \text { if } \beta-\gamma>\gamma>0 \\ f^{(d)} \mid V_{2}=\lambda f^{(d)} & \text { if } \beta=2 \gamma\end{cases}
$$

(ii) If $\alpha-\beta=1$, then

$$
\begin{cases}\left(g^{(d)}+\lambda g^{(2 d)}\right) \mid V_{2}=-\left(g^{(d)}+\lambda g^{(2 d)}\right) & \text { if } \gamma=0, \\ g^{(d)} \mid V_{2}=+g^{(d)} & \text { if } \beta-\gamma>\gamma>0, \\ f^{(d)} \mid V_{2}=\lambda f^{(d)} & \text { if } \beta=2 \gamma .\end{cases}
$$

Proof. Write $S=S_{2}$ and $W=W_{2^{\alpha}}^{(N)}$. Since $S \tau=\tau+1 / 2$, we have $f^{(d)} \mid S=+f^{(d)}$ if $\gamma>0$, and $f^{(d)} \mid S=-f^{(d)}$ if $\alpha-\beta \geq 2$ and $\gamma=0$ (note that if $\alpha-\beta \geq 2$, then $a_{2 m}=0$ for $m=1,2, \ldots$ ). The assertions, except for the case $\alpha-\beta=1$ and $\gamma=0$, follow from these and Lemma 1. Finally, let $\alpha-\beta=1$ and $\gamma=0$. Then

$$
f^{(d)}+f^{(d)} \mid S=2 \sum_{n=1}^{\infty} a_{2 n} q^{2 d n}=2 a_{2} \sum_{n=1}^{\infty} a_{n} q^{2 d n}=2 a_{2} f^{(2 d)}=-2 \lambda f^{(2 d)},
$$

so we have

$$
f^{(d)}\left|V_{2}=-2 \lambda f^{(2 d)}\right| W S-f^{(d)} \mid W S=-2^{\beta-1} f^{\left(2^{\beta-1} d\right)}-2^{\beta} \lambda f^{\left(2^{\beta} d\right) .}
$$

From this, we see that

$$
\begin{aligned}
\left(f^{(d)}-f^{(d)} \mid V_{2}\right)+\lambda & \left(f^{(2 d)}-f^{(2 d)} \mid V_{2}\right) \\
& =\left(f^{(d)}+2^{\beta} \lambda f^{\left(2^{\beta} d\right)}\right)+\lambda\left(f^{(2 d)}+2^{\beta-2} \lambda f^{\left(2^{\beta-1} d\right)}\right),
\end{aligned}
$$

hence the assertion follows.

Proposition 3. Let $N=9 M$ with $M$ a positive integer such that $3 \nmid M$. Let $M^{\prime}$ be a positive divisor of $M$, and let d be a positive divisor of $M / M^{\prime}$.

(i) Let $f=\sum a_{n} q^{n}$ be a newform on $\Gamma_{0}\left(9 M^{\prime}\right)$ such that $f \mid W_{9}^{\left(9 M^{\prime}\right)}=$ $+f$. Then $f^{(d)}$ is an eigenform of $V_{3}$ with eigenvalue +1 .

(ii) Let $f=\sum a_{n} q^{n}$ be a newform on $\Gamma_{0}\left(3 M^{\prime}\right)$ such that $f \mid W_{3}^{\left(3 M^{\prime}\right)}=\lambda f$. Then $f^{(d)}+3 \lambda f^{(3 d)}$ is an eigenform of $V_{3}$ with eigenvalue -1 .

Proof. Write $S=S_{3}$ and $W=W_{9}^{(9 M)}$, and put $\zeta=\exp (2 \pi i / 3)$. Since $S \tau=\tau+1 / 3$, it follows that

$$
\begin{aligned}
f^{(d)}+f^{(d)}\left|S+f^{(d)}\right| S^{2} & =\sum\left(1+\zeta^{d n}+\zeta^{2 d n}\right) a_{n} q^{d n} \\
& =3 \sum a_{3 n} q^{3 d n}=3 a_{3} \sum a_{n} q^{3 d n} .
\end{aligned}
$$

(i) In this case, we have

$$
\left(f^{(d)}\left|S+f^{(d)}\right| S^{2}\right)\left|W=f^{(d)}\right| S+f^{(d)} \mid S^{2},
$$


since $a_{3}=0$ and $f \mid W_{9}^{\left(9 M^{\prime}\right)}=f$. On the other hand, by Theorem 6 of [1],

$$
g:=\frac{1}{\sqrt{-3}}\left(f|S-f| S^{2}\right)=\sum_{n=1}^{\infty}\left(\frac{-3}{n}\right) a_{n} q^{n}
$$

is also a newform on $\Gamma_{0}\left(9 M^{\prime}\right)$ with $g \mid W_{9}^{\left(9 M^{\prime}\right)}=g$. Hence $f^{(d)}\left|S W=f^{(d)}\right| S$, or equivalently, $f^{(d)} \mid S W S^{2}=f^{(d)}$.

(ii) In this case, we have

$$
f^{(d)}+f^{(d)}\left|S+f^{(d)}\right| S^{2}=3 a_{3} f^{(3 d)}=-f^{(d)} \mid W,
$$

so

$$
f^{(d)}\left|W S^{2}+f^{(d)}\right| S W S^{2}+f^{(d)}\left|S^{2} W S^{2}=-f^{(d)}\right| S^{2} .
$$

Now we have

$$
3 \lambda f^{(3 d)}\left|S W S^{2}=3 \lambda f^{(3 d)}\right| W S^{2}=f^{(d)} \mid S^{2}
$$

and

$$
f^{(d)}\left|W S^{2}=3 \lambda f^{(3 d)}\right| S^{2}=3 \lambda f^{(3 d)} .
$$

Also we compute

$$
f^{(d)}\left|S^{2} W S^{2}=f^{(d)}\right| W S W=3 \lambda f^{(3 d)}\left|S W=3 \lambda f^{(3 d)}\right| W=f^{(d)},
$$

since $(W S)^{3} \in \Gamma_{0}(9 M)$. Hence we obtain the equality

$$
\left(f+3 \lambda f^{(3)}\right) \mid S W S^{2}=-\left(f+3 \lambda f^{(3)}\right),
$$

as desired.

As a consequence of these results, we can determine the hyperellipticity of $X_{0}^{*}(N)$ for some cases.

Remark 2. To calculate $\bar{g}$, it seems more natural to find the formula for the number of the fixed points for $V$, but our method has the advantage of giving the defining equation of $X_{0}^{*}(N)$ (see also the last section).

Example 1 . Let $N=136=8 \cdot 17$. Then $S_{2}^{*}(136)$ is spanned by

$$
f_{1}-4 f_{1}^{(4)}, \quad f_{2}-2 f_{2}^{(2)}, \quad f_{3}-2 f_{3}^{(2)}
$$

where $f_{1}, f_{2}, f_{3}$ are newforms such that $f_{1} \in S_{2}^{0}(2 \cdot 17)^{(-,+)}$and $f_{2}, f_{3} \in$ $S_{2}^{0}(4 \cdot 17)^{(-,+)}$. Here and in what follows, signatures indicate the eigenvalues of Atkin-Lehner involutions (see [2]). Thus $X_{0}^{*}(136) /\left\langle V_{2}\right\rangle \cong \mathbb{P}^{1}$, from which we see that $X_{0}^{*}(136)$ is hyperelliptic, with hyperelliptic involution $V_{2}$. 
EXAmple 2. Let $N=360=8 \cdot 45$. A basis $\left\langle g_{1}, \ldots, g_{7}\right\rangle$ of $S_{2}^{*}(360)$ is given by

$$
\begin{aligned}
& g_{1}=f_{1}-2 f_{1}^{(2)}+9 f_{1}^{(9)}-18 f_{1}^{(18)}, \\
& g_{2}=f_{1}^{(3)}-2 f_{1}^{(6)}, \\
& g_{3}=f_{2}+2 f_{2}^{(2)}+4 f_{2}^{(4)}-3 f_{2}^{(3)}-6 f_{2}^{(6)}-12 f_{2}^{(12)}, \\
& g_{4}=f_{2}^{(2)}-3 f_{2}^{(6)} \\
& g_{5}=f_{3}-2 f_{3}^{(2)}+5 f_{3}^{(5)}-10 f_{3}^{(10)}, \\
& g_{6}=f_{4}-4 f_{4}^{(4)} \\
& g_{7}=f_{5}-3 f_{5}^{(3)},
\end{aligned}
$$

where $f_{1} \in S_{2}^{0}(4 \cdot 5)^{(-,+)}, f_{2} \in S_{2}^{0}(2 \cdot 3 \cdot 5)^{(+,-,+)}, f_{3} \in S_{2}^{0}(4 \cdot 9)^{(-,+)}, f_{4} \in$ $S_{2}^{0}(2 \cdot 9 \cdot 5)^{(-,+,+)}$and $f_{5} \in S_{2}^{0}(8 \cdot 3 \cdot 5)^{(+,-,+)}$. Then, by Proposition 2 , we see that $S_{2}^{*}(360)^{V_{2}}=\left\langle g_{4}, g_{7}\right\rangle_{\mathbb{C}}$, hence the genus $\bar{g}=\bar{g}\left(360 ; V_{2}\right)$ of $X_{0}^{*}(360) /\left\langle V_{2}\right\rangle$ is 2 . Therefore $X_{0}^{*}(360)$ is not hyperelliptic, by the following proposition.

Proposition 4. Let $X / \mathbb{C}$ be a hyperelliptic curve of genus $g$. Let $w$ be an involution on $X$, and $\bar{g}$ the genus of $X /\langle w\rangle$. Suppose $\bar{g} \neq 0$. If $g$ is even, then $\bar{g}=g / 2$, and if $g$ is odd, then $\bar{g}=(g+1) / 2$ or $(g-1) / 2$.

Proof. This is a corollary to Proposition 1 of [12], whose statement will be given in the next section.

EXAmPle 3. Put $\alpha_{n}:=\left(\begin{array}{ll}n & 0 \\ 0 & 1\end{array}\right)$. An easy calculation shows that

$$
\alpha_{n} \Gamma_{0}^{*}\left(n^{2} N\right) \alpha_{n}^{-1} \subseteq \Gamma_{0}^{*}(N) .
$$

Let $n=2$. Then $\alpha_{2} \Gamma_{0}^{*}(8 N) \alpha_{2}^{-1}$ is of index 4 in $\Gamma_{0}^{*}(2 N)$. Now consider the curve $X_{0}^{*}(840)$, which is of genus 10 . Then there is a covering $X_{0}^{*}(840) \rightarrow$ $X_{0}^{*}(210)$ of degree 4 . We can regard this covering as a composition

$$
X_{0}^{*}(840) \rightarrow X^{\prime} \rightarrow X_{0}^{*}(210)
$$

of coverings of degree 2 . Therefore, by the above proposition, we conclude that $X_{0}^{*}(840)$ is not hyperelliptic, since $X_{0}^{*}(210)$ is of genus 1 (see [2]).

By the same reason, $X_{0}^{*}(1680)$ is not hyperelliptic, since $X_{0}^{*}(1680)$ is of genus 19 and $X_{0}^{*}(420)$ is of genus 3 .

To calculate $\bar{g}(N ; V)$ in this way, we need information on the $W$-splitting of $S_{2}^{0}(N)$. For $N \leq 300$, such data are given in [2]. For $N \geq 301$, we include here a table of the $W$-splitting of $S_{2}^{0}(N)$ to the extent of our needs. (Remark. The third column of Table 2 gives dimensions of direct summands $S_{2}^{0}(N)^{( \pm, \ldots, \pm)}$ of $S_{2}^{0}(N)$, ordered lexicographically.) 
Table 2. The $W$-splitting of $S_{2}^{0}(N)$

\begin{tabular}{ccc}
\hline$N$ & $p \mid N$ & $\begin{array}{c}\text { The } W \text {-splitting } \\
\text { of } S_{2}^{0}(N)\end{array}$ \\
\hline 306 & $2,3,17$ & $0,2,1,1,2,0,0,2$ \\
312 & $2,3,13$ & $1,1,1,0,1,0,1,1$ \\
315 & $3,5,7$ & $2,0,2,0,2,1,0,3$ \\
336 & $2,3,7$ & $0,1,1,0,1,1,0,2$ \\
342 & $2,3,19$ & $1,0,2,1,1,0,0,2$ \\
\hline
\end{tabular}

\begin{tabular}{ccc}
\hline$N$ & $p \mid N$ & $\begin{array}{c}\text { The } W \text {-splitting } \\
\text { of } S_{2}^{0}(N)\end{array}$ \\
\hline 360 & $2,3,5$ & $0,1,1,0,1,0,1,1$ \\
396 & $2,3,11$ & $0,0,0,0,0,0,1,2$ \\
450 & $2,3,5$ & $1,0,2,1,1,0,0,2$ \\
630 & $2,3,5,7$ & $0,1,1,0,1,1,1,1$ \\
& & $1,0,0,1,0,1,1,0$ \\
\hline
\end{tabular}

Table 3. Genera of $X_{0}^{*}(N)$ and $X_{0}^{*}(N) /\langle V\rangle$

\begin{tabular}{cccc}
\hline & & Genus of & Genus of \\
& $V$ & $X_{0}^{*}(N)$ & $X_{0}^{*}(N) /\langle V\rangle$ \\
\hline 136 & $V_{2}$ & 3 & 0 \\
144 & $V_{2}$ & 3 & 1 \\
& $V_{3}$ & 3 & 1 \\
& $V_{2} V_{3}$ & 3 & 1 \\
152 & $V_{2}$ & 3 & 1 \\
171 & $V_{3}$ & 3 & 0 \\
207 & $V_{3}$ & 3 & 2 \\
234 & $V_{3}$ & 3 & 1 \\
240 & $V_{2}$ & 3 & 1 \\
252 & $V_{3}$ & 3 & 0 \\
312 & $V_{2}$ & 3 & 1 \\
315 & $V_{3}$ & 3 & 2 \\
160 & $V_{2}$ & 4 & 2 \\
\hline
\end{tabular}

\begin{tabular}{cccc}
\hline$N$ & $V$ & $\begin{array}{c}\text { Genus of } \\
X_{0}^{*}(N)\end{array}$ & $\begin{array}{c}\text { Genus of } \\
X_{0}^{*}(N) /\langle V\rangle\end{array}$ \\
\hline 176 & $V_{2}$ & 4 & 0 \\
264 & $V_{2}$ & 4 & 1 \\
280 & $V_{2}$ & 4 & 1 \\
306 & $V_{3}$ & 4 & 1 \\
342 & $V_{3}$ & 4 & 1 \\
216 & $V_{2}$ & 5 & 2 \\
279 & $V_{3}$ & 5 & 0 \\
396 & $V_{3}$ & 5 & 3 \\
630 & $V_{3}$ & 5 & 2 \\
336 & $V_{2}$ & 6 & 2 \\
360 & $V_{2}$ & 7 & 2 \\
450 & $V_{3}$ & 7 & 3 \\
& & & \\
\hline
\end{tabular}

We know from Table 3 that $X_{0}^{*}(N)$ is hyperelliptic with hyperelliptic involution $V$ for $N=136,171,252,176$ and 279. We also know that $X_{0}^{*}(N)$ is not hyperelliptic for $N=264,280,306,342,336$ and 360, by virtue of Proposition 4. Further, we use the following fact to conclude that $X_{0}^{*}(207)$ and $X_{0}^{*}(315)$ are hyperelliptic.

Proposition 5. Let $X, Y$ be curves over $\mathbb{C}$ of genus 3, 2, respectively. If there is a covering $\pi: X \rightarrow Y$, then $X$ is hyperelliptic.

Proof. See $[6]$.

Determination of the hyperellipticity of $X_{0}^{*}(N)$ for $N=144,152,234$, $240,312,160,216,396,630$ and 450 will be postponed to the following sections.

3. Fixed points of $V_{2}$. In this section, we always assume that $8 \mid N$ except for Remark 3. The important fact is that $V_{2}$ is defined over $\mathbb{Q}$, so that the following Ogg's observations [12] are applicable. 
Lemma 2 ([12], Prop. 1$)$. Let $X / \mathbb{C}$ be a hyperelliptic curve, and $v$ the hyperelliptic involution on $X$. Let $w$ be another involution, and put $u=v w$, which is also an involution. Then the fixed-point sets of $u, v$, and $w$ are disjoint. If $g$ is even, then $w$ and $u$ have two fixed points each. If $g$ is odd, then $w$ has four fixed points, and u has none, or vice versa.

Proposition 6. Let $X$ be a curve defined over $\mathbb{Q}$, and let $w$ be a nonhyperelliptic involution defined over $\mathbb{Q}$ on $X$. If $w$ has only one rational fixed point, then $X$ is non-hyperelliptic.

Proof. Suppose $X$ is hyperelliptic. Then the hyperelliptic involution $v$, which is in the center of Aut $X$, acts on the set of fixed points of $w$. But $v$ is defined over $\mathbb{Q}$, so $v$ must fix the (unique) rational fixed point of $w$. This is a contradiction.

Observe that $S_{2}=\left(\begin{array}{ll}2 & 1 \\ 0 & 2\end{array}\right)$ commutes with all $W_{p^{\nu}}, p^{\nu} \| N, p \neq 2$. Therefore $S_{2}$ induces an isomorphism

$$
X_{0}^{*}(N) \cong X_{0}(N) / G,
$$

where $G$ is the subgroup of Aut $X_{0}(N)$ generated by $\left\{W_{p^{\nu}}\right\}_{p \neq 2} \cup\left\{V_{2}\right\}$. Thus, to obtain the information about the fixed points of $V_{2}$ on $X_{0}^{*}(N)$, it suffices to consider the fixed points of $W_{2^{\alpha}}\left(2^{\alpha} \| N\right)$ on $X_{0}(N) / G$.

EXAmple 4 . Let $N=152=2^{3} \cdot 19$. The genus of $X_{0}^{*}(152)$ is three, and $V_{2}$ has 4 fixed points on $X_{0}^{*}(152)$ (see Table 3 ). Put $G=\left\langle W_{19}, V_{2}\right\rangle$, which is abelian of type $(2,2)$. On $X_{0}(152)$, we can see from [2] that $W_{8}$ and $W_{152}$ have 4 and 12 fixed points, respectively. Since $G$ acts fixed-point-freely on the fixed-point set of $W_{8}$ (resp. $W_{152}$ ), the contribution of the fixed points of $W_{8}$ (resp. $\left.W_{152}\right)$ on $X_{0}(152)$ to those of $W_{8}$ on $X=X_{0}(152) / G$ is one (resp. three). Further we have

$$
h(-4 \cdot 8)=2, \quad h(-4 \cdot 152)=12,
$$

where $h(-d)$ is the class number of primitive quadratic forms of discriminant $-d$. This means that the fixed points of $W_{152}$ are all defined over a field of degree exactly 12 . Hence $W_{8}$ has only one rational fixed point on $X$, i.e., $X_{0}^{*}(152) \cong X$ is not hyperelliptic.

The similar argument can be applied to the cases $N=216$ and 312. Let $N=216$. The genus of $X_{0}^{*}(216)$ is five, and the involution $V_{2}$ has four fixed points on $X_{0}^{*}(216)$. On $X_{0}(216)$, we see from [2] that $W_{8}$ (resp. $\left.W_{216}\right)$ has 4 (resp. 12) fixed points. Also we compute $h(-4 \cdot 8)=2, h(-4 \cdot 216)=12$. Hence $X_{0}^{*}(216)$ is not hyperelliptic.

For $N=312$, we include the $W$-splitting of $S_{2}(312)$ :

$$
3,10,7,5,6,6,9,3,
$$


which should be read in the same manner as the data in the third column of Table 2. From this, we see that $W_{104}$ has 24 fixed points and $W_{312}$ has 8 fixed points; the corresponding class numbers are $h(-4 \cdot 104)=12, h(-4 \cdot 312)=8$. Therefore $W_{8}$ has exactly one rational fixed point on $X_{0}(312) / G$, where $G=\left\langle W_{3}, W_{13}, V_{2}\right\rangle$. Hence $X_{0}^{*}(312)$ is not hyperelliptic.

Remark 3. Let $9 \| N$, and let $V_{3}$ be the involution on $X_{0}(N)$ defined in the previous section. Then $V_{3}$ is defined over $\mathbb{Q}(\sqrt{-3})$. Also, it can easily be shown that

$$
V_{3} W_{p^{\nu}} V_{3}= \begin{cases}W_{p^{\nu}} & \text { if } p^{\nu} \equiv 0,1(\bmod 3), \\ W_{9} W_{p^{\nu}} & \text { if } p^{\nu} \equiv 2(\bmod 3)\end{cases}
$$

for $p^{\nu} \| N$ (cf. Theorem 8 of [1]).

4. Modular involutions on $X_{0}^{*}(N)$ (II). In this section, we assume that $4 \| N$. Write $N=4 M$. Then

Proposition 7. We have the isomorphism

$$
X_{0}^{*}(N) \cong X_{0}(N) / G \cong X_{0}(2 M) /\left\langle\left\{W_{p^{\nu}}\right\}_{p \mid M}\right\rangle,
$$

where $G$ is a subgroup of Aut $X_{0}(N)$ generated by $\left\{W_{p^{\nu}}\right\}_{p \neq 2} \cup\left\{S_{2}\right\}$.

Proof. Indeed, the first isomorphism is obtained by conjugating $\Gamma_{0}^{*}(N)$ by $S_{2} W_{4} S_{2}$, and the second by conjugating $\left\langle G \cup \Gamma_{0}(N)\right\rangle$ by $\alpha_{2}$.

Consider the case $N=300$. Then we have

$$
X_{0}^{*}(300) \cong X_{0}(150) /\left\langle W_{3}, W_{25}\right\rangle,
$$

the right hand side of which has a covering of degree two to $X_{0}^{*}(150)$; since the genus of $X_{0}^{*}(300)$ is four and that of $X_{0}^{*}(150)$ is one, we find that $X_{0}^{*}(300)$ is not hyperelliptic (Proposition 4).

Next we pick up the case $N=348$. Then

$$
X_{0}^{*}(348) \cong X:=X_{0}(174) /\left\langle W_{3}, W_{29}\right\rangle .
$$

The curve $X$ has an involution induced by $W_{2}$. Since (on $\left.X_{0}(174)\right) W_{6}$ and $W_{174}$ have 4 and 12 fixed points each, and since $h(-4 \cdot 6)=2$ and $h(-4 \cdot 174)=12$, it follows that $W_{2}$ has only one rational fixed point, as in Example 4 . So, by Proposition 6, we conclude that $X_{0}^{*}(348)$ is not hyperelliptic.

5. Reduction modulo $p$. Let $p$ be a prime number and $N$ a positive integer such that $N=p M, p \nmid M$. The reduction modulo $p$ of $X_{0}(p M)$ consists of two copies $Z, Z^{\prime}$ of $X_{0}(M)$ in characteristic $p$, intersecting transversally at the supersingular points [3] (see Figure 1). 


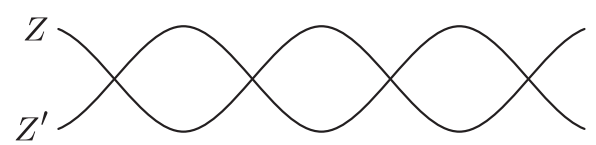

Fig. 1. $X_{0}(p M) \bmod p$

The Atkin-Lehner involutions $W_{N^{\prime}}\left(N^{\prime} \| N\right)$ still act on $X_{0}(N) \bmod p$. If $p \nmid N^{\prime}$, then $W_{N^{\prime}}$ fixes each component $Z, Z^{\prime}$, and its action in characteristic $p$ is the same as in characteristic 0 . If $p \mid N^{\prime}$, then $W_{N^{\prime}}$ interchanges $Z$ and $Z^{\prime}$. In particular, if $N^{\prime}=p$, then $W_{p}$ fixes each $\mathbb{F}_{p}$-rational supersingular point, while it exchanges each properly $\mathbb{F}_{p^{2}}$-rational supersingular point for its conjugate. Let $W^{\prime}$ be a subgroup of $W(N)$. If $W^{\prime}$ is generated by some of $W_{N^{\prime}}$ with $p \nmid N^{\prime}$, then $X_{0}(N) / W^{\prime} \bmod p$ is again of the shape in Figure 1 with $Z=Z^{\prime}=X_{0}(M) / W^{\prime}$. If $W^{\prime}$ contains some $W_{N^{\prime}}$ with $p \mid N^{\prime}$, then $X_{0}(N) / W^{\prime} \bmod p$ becomes as in Figure 2 :

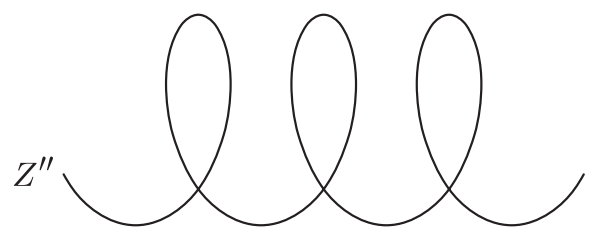

Fig. 2

where $Z^{\prime \prime}=X_{0}(M) / W^{\prime \prime}$ is some quotient of $X_{0}(M)$.

Now assume that $X_{0}(N) / W^{\prime}$ is hyperelliptic. Assume further for simplicity that the special fibre of the minimal model of $X_{0}(N) / W^{\prime}$ at $p$ is as in Figure 2, with $X_{0}(N) / W^{\prime \prime}$ being of genus zero. Then, as explained in Appendix $\mathrm{C}$ of [7], there must exist an element $A$ of order 2 of $\mathrm{PGL}_{2}\left(\mathbb{F}_{p}\right)$ such that

$$
A \alpha=\bar{\alpha}
$$

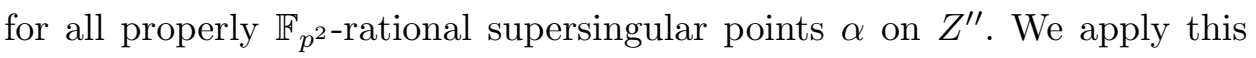
observation to the cases $N=164$ and 234 .

Let $N=164=41 \cdot 4$. We have $X_{0}^{*}(164) \cong X_{0}(82) /\left\langle W_{41}\right\rangle=: X$ by Proposition 7. Consider $X_{0}(82)$ modulo $p=41$. Then $Z=Z^{\prime}=X_{0}(2)$ is of genus zero, and the curve $X_{0}(2)$ is defined by the equation

$$
j=64 \frac{(x+4)^{3}}{x^{2}}
$$

(see [4]). Since the supersingular $j$-invariants in characteristic $p=41$ are given by

$$
j(j+38)(j+13)(j+9)=0,
$$

we compute the supersingular points on $X_{0}(2)$ in characteristic $p=41$ by solving the equation (2):

$(x+4)\left[(x+31)\left(x^{2}+29 x+10\right)\right]\left[(x+23)\left(x^{2}+27 x+1\right)\right]\left[(x+25)\left(x^{2}+7 x+37\right)\right]=0$, 
where $x=-4$ is the only supersingular point above $j=0$. Thus the special fibre of the minimal model of $X$ at $p=41$ is as in Figure 2, with $Z^{\prime \prime}=$ $X_{0}(2)$. But it can easily be checked that there does not exist an element of order 2 of $\mathrm{PGL}_{2}\left(\mathbb{F}_{41}\right)$ with the property (1), from which we conclude that $X_{0}^{*}(164) \cong X$ is not hyperelliptic.

Let $N=234=13 \cdot 18$, and consider $X_{0}(234)$ modulo $p=13$. Then $Z=Z^{\prime}=X_{0}(18)$ is of genus zero, and the curve $X_{0}(18)$ is defined by the equation

$$
j=h \circ g \circ f(x),
$$

where

$$
\begin{aligned}
f(X) & =\frac{1}{2} X\left(X^{2}+6 X+12\right), \\
g(X) & =\frac{X(2 X+9)^{2}}{27(X+4)}, \\
h(X) & =27 \frac{(X+1)(9 X+1)^{3}}{X} .
\end{aligned}
$$

The actions of $W_{2}, W_{9}, W_{18}$ are given by

$$
x\left|W_{2}=\frac{-2(x+3)}{x+2}, \quad x\right| W_{9}=\frac{-3(x+2)}{x+3}, \quad x \mid W_{18}=\frac{6}{x}
$$

(cf. [4]). The only supersingular $j$-invariant in characteristic $p=13$ is $j=5$, and the supersingular points on $X_{0}(18)$ are obtained by solving the equation $(3)$ :

$$
\begin{aligned}
\left(x^{2}+\right. & 6)\left(x^{2}+7\right)\left(x^{2}+x+5\right)\left(x^{2}+3 x+10\right)\left(x^{2}+4 x+6\right)\left(x^{2}+4 x+9\right) \\
& \times\left(x^{2}+4 x+10\right)\left(x^{2}+5 x+1\right)\left(x^{2}+5 x+5\right)\left(x^{2}+6 x+2\right)\left(x^{2}+6 x+4\right) \\
& \times\left(x^{2}+7 x+1\right)\left(x^{2}+7 x+4\right)\left(x^{2}+8 x+10\right)\left(x^{2}+9 x+2\right)\left(x^{2}+9 x+9\right) \\
& \times\left(x^{2}+10 x+1\right)\left(x^{2}+10 x+6\right)=0 .
\end{aligned}
$$

Put $X=X_{0}^{*}(234)$ and consider $X$ modulo $p=13$, which is of the shape in Figure 2 with $Z^{\prime \prime}=X_{0}^{*}(18)$. The curve $X_{0}^{*}(18)$ is parametrized by

$$
x^{\prime}=x+x\left|W_{2}+x\right| W_{9}+x \mid W_{18}=\frac{\left(x^{2}-6\right)^{2}}{x(x+2)(x+3)} .
$$

There are three conjugate pairs of properly $\mathbb{F}_{13^{2}}$-rational supersingular points, say, $\alpha_{i}, i=1, \ldots, 6$, with $\alpha_{i+3}=\bar{\alpha}_{i}$ for $i=1,2,3$; they are the roots of the equation

$$
\left(x^{\prime 2}-6 x^{\prime}+7\right)\left(x^{\prime 2}-6 x^{\prime}-9\right)\left(x^{2}-32\right)=0 .
$$

It can be shown that the number of the points on $Z=Z^{\prime}$ above each of the three conjugate pairs is equal to the degree of the covering $X_{0}(238) \rightarrow X$. Hence the special fibre of the minimal model of $X$ at $p=13$ is of the shape 
in Figure 2, with $Z^{\prime \prime}=X_{0}^{*}(18)$. But there does not exist an element of order 2 of $\mathrm{PGL}_{2}\left(\mathbb{F}_{13}\right)$ with the property (1), from which we conclude that $X_{0}^{*}(234)$ is not hyperelliptic.

6. Gap sequences. Let $X$ be an algebraic curve over $\mathbb{C}$ of genus $g$. The Weierstrass gap sequence $G_{P}$ at a point $P$ of $X$ is defined by

$$
G_{P}=\left\{n \in \mathbb{Z} \mid n>0 \text { and }(f)_{\infty} \neq n(P) \text { for all } f \in K(X)\right\},
$$

where $K(X)$ is the function field of $X$ and $(f)_{\infty}$ is the polar divisor of $f$. By the Riemann-Roch theorem, we see that the cardinality of $G_{P}$ is exactly the genus $g$ of $X$ with $\max G_{P} \leq 2 g-1$. It is easily shown that

$$
G_{P}=\left\{n \in \mathbb{Z} \mid \exists \omega \in H^{0}\left(X, \omega_{X}\right) \text { such that } \operatorname{ord}_{P}(\omega)=n-1\right\},
$$

where $\boldsymbol{\omega}_{X}$ is the canonical sheaf on $X$. A point $P$ on $X$ is a Weierstrass point if $G_{P} \neq\{1,2, \ldots, g\}$. If $X$ is hyperelliptic and $P$ is a Weierstrass point of $X$, then

$$
G_{P}=\{1,3,5, \ldots, 2 g-1\} .
$$

Now let $\Gamma$ be a Fuchsian group of the first kind having $i \infty$, the point at infinity, as its cusp. For simplicity, we assume that the local parameter at the point $i \infty$ is $q=\exp (2 \pi i \tau)$. Let $S_{2}(\Gamma)$ be the space of cuspforms of weight 2 on $\Gamma$. If the corresponding algebraic curve $X=X_{\Gamma}$ is of genus $g$, then $\operatorname{dim}_{\mathbb{C}} S_{2}(\Gamma)=g$. Since $S_{2}(\Gamma)$ can be identified with the space of holomorphic 1-forms on $X$ by sending $f$ to $2 \pi i f d \tau$, we can interpret (4) as

$$
G_{P}=\left\{n \in \mathbb{Z} \mid \exists f \in S_{2}(\Gamma) \text { such that } f=q^{n}+\ldots\right\} .
$$

Hence if $X$ is hyperelliptic, then there is a basis $\left\langle f_{1}, \ldots, f_{g}\right\rangle$ of $S_{2}(\Gamma)$ of the form

$$
\left\{\begin{array}{l}
f_{1}=q+a_{2}^{(1)} q^{2}+a_{3}^{(1)} q^{3}+\ldots \\
f_{2}=q^{2}+a_{3}^{(2)} q^{3}+\ldots \\
\ldots \ldots \ldots \ldots \ldots \ldots \ldots \ldots \ldots \ldots \ldots \ldots \ldots \\
f_{g}=q^{g}+a_{g+1}^{(g)} q^{g+1}+\ldots
\end{array}\right.
$$

or

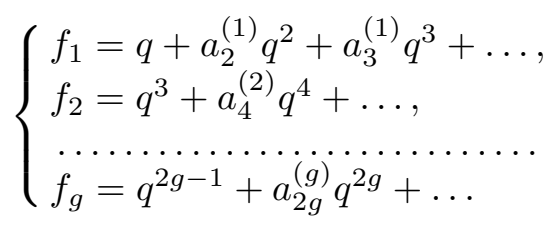

according as $\overline{i \infty}$ is an ordinary point or a Weierstrass point. In other words, if $S_{2}(\Gamma)$ does not have a basis of the form (6) nor (7), then $X$ is not hyperelliptic. In the following, $\Gamma$ is taken to be some normalizer of $\Gamma_{0}(N)$, and we use trace formulas of Hecke operators to compute Fourier coefficients of $f_{1}, \ldots, f_{g}([8],[13])$. 
EXAmple 5. Let $N=160$. Then $X_{0}^{*}(160)$ is of genus 4 , so $S_{2}^{*}(160)$ is of dimension 4 . On the other hand, $S_{2}^{*}(160)$ contains

$$
\begin{aligned}
f_{1}-8 f_{1}^{(8)} & =q-2 q^{3}-q^{5}+2 q^{7}+\ldots, \\
f_{1}^{(2)}-2 f_{1}^{(4)} & =q^{2}-2 q^{4}-2 q^{6}-q^{10}+\ldots, \\
f_{2} & =q-2 q^{3}-q^{5}-2 q^{7}+\ldots,
\end{aligned}
$$

where $f_{1} \in S_{2}^{0}(4 \cdot 5)^{(-,+)}$and $f_{2} \in S_{2}^{0}(32 \cdot 5)^{(+,+)}$. Hence $S_{2}^{*}(160)$ cannot have a basis of the form (6) nor (7). Namely, $X_{0}^{*}(160)$ is not hyperelliptic.

By the same reason, $X_{0}^{*}(175)$ and $X_{0}^{*}(270)$ are not hyperelliptic; $X_{0}^{*}(175)$ is of genus 3 and $S_{2}^{*}(175)$ contains

$$
f_{1}-5 f_{1}^{(5)}=q-q^{3}+2 q^{4}+\ldots, \quad f_{2}=q-q^{3}-2 q^{4}+\ldots,
$$

where $f_{1} \in S_{2}^{0}(5 \cdot 7)^{(-,+)}$and $f_{2} \in S_{2}^{0}(25 \cdot 7)^{(+,+)} ; X_{0}^{*}(270)$ is of genus 3 and $S_{2}^{*}(270)$ is spanned by

$$
\begin{aligned}
& g_{1}:=f_{1}-9 f_{1}^{(9)}=q-q^{2}+q^{3}+q^{4}+\ldots, \\
& g_{2}:=f_{2}+2 f_{2}^{(2)}-3 f_{2}^{(3)}-6 f_{2}^{(6)}=q+3 q^{2}-3 q^{3}+q^{4}+\ldots, \\
& g_{3}:=f_{3}+2 f_{3}^{(2)}=q-2 q^{4}+\ldots,
\end{aligned}
$$

where $f_{1} \in S_{2}^{0}(2 \cdot 3 \cdot 5)^{(+,-,+)}, f_{2} \in S_{2}^{0}(9 \cdot 5)^{(-,+)}$and $f_{3} \in S_{2}^{0}(27 \cdot 5)^{(+,+)}$, so

$$
3 g_{1}+g_{2}-4 g_{3}=12 q^{4}+\ldots \in S_{2}^{*}(270) \text {. }
$$

ExAmple 6 . Let $N=396$. Then $X_{0}^{*}(396) /\left\langle V_{3}\right\rangle$ is of genus 3 , and the space $S_{2}^{*}(396)^{V_{3}}$ is spanned by $f_{1}+4 f_{1}^{(4)}, f_{1}^{(2)}$ and $f_{2}-2 f_{2}^{(2)}$, where $f_{1} \in$ $S_{2}^{0}(9 \cdot 11)^{(+,+)}$and $f_{2} \in S_{2}^{0}(2 \cdot 9 \cdot 11)^{(-,+,+)}$(see [2] and Proposition 3). But since their levels are divisible by $3^{2}, f_{1}$ and $f_{2}$ have zero as their third Fourier coefficients, implying that $S_{2}^{*}(396)^{V_{3}}$ cannot have a basis of the form (6) nor (7). This shows that $X_{0}^{*}(396) /\left\langle V_{3}\right\rangle$ is not hyperelliptic. Hence $X_{0}^{*}(396)$ is also non-hyperelliptic.

By the same reason, $X_{0}^{*}(450)$ is not hyperelliptic (the space $S_{2}^{*}(450)^{V_{3}}$ is spanned by $f_{1}-5 f_{1}^{(5)}, f_{2}+2 f_{2}^{(2)}$ and $f_{3}$, where $f_{1} \in S_{2}^{0}(2 \cdot 9 \cdot 5)^{(+,+,-)}$, $f_{2} \in S_{2}^{0}(9 \cdot 25)^{(+,+)}$and $\left.f_{3} \in S_{2}^{0}(2 \cdot 9 \cdot 25)^{(+,+,+)}\right)$.

7. Conclusion: Remaining cases and the defining equations of hyperelliptic curves $X_{0}^{*}(N)$. So far, we have determined the hyperellipticity of $X_{0}^{*}(N)$ except for the following values of $N$ :

$$
N=144,162,196,240,294,420,476,630 .
$$

We are now going to treat these remaining cases. Let $\Gamma$ be as in the previous section, and $\left\langle f_{1}, \ldots, f_{g}\right\rangle$ a basis of $S_{2}(\Gamma)$. Assume for simplicity 
that $\overline{i \infty}$ is an ordinary point of $X_{\Gamma}$, and that $\left\langle f_{1}, \ldots, f_{g}\right\rangle$ is of the form (6). Put

and define

$$
z=\frac{f_{g-1}}{f_{g}}, \quad w=\frac{d z}{2 \pi i f_{g} d \tau}=\left(\frac{f_{g}}{q}\right)^{-1} \frac{d z}{d q}
$$

$$
G(T)=T^{2 g+2}+v_{2 g+1} T^{2 g+1}+\ldots+v_{0} \in \mathbb{C}[T]
$$

by the condition $\operatorname{ord}_{q}\left(w^{2}-G(z)\right) \geq 1$, i.e., the Laurent series $w^{2}-G(z)$ consists only of positive $q$-power terms. Thus we can write

$$
w^{2}-G(z)=\sum_{j \geq 1} d_{j} q^{j} .
$$

Proposition 8. Notation being as above, the curve $X=X_{\Gamma}$ is hyperelliptic if and only if the following two conditions hold:

(i) $G(T)$ is separable,

(ii) $d_{1}=\ldots=d_{h}=0$ where $h=4 g^{2}+8 g-20$.

Moreover, if $X$ is hyperelliptic, then it is defined by the equation $w^{2}=G(z)$.

Proof. See [7].

Example 7 . Let $N=144$. Then a basis of $S_{2}^{*}(144)$ is given by

$$
\begin{aligned}
& f_{1}=q-4 q^{4}-4 q^{7}+2 q^{13}+O\left(q^{17}\right), \\
& f_{2}=q^{2}-4 q^{4}+2 q^{5}+2 q^{6}-4 q^{7}+2 q^{9}-2 q^{10}+4 q^{13}+O\left(q^{17}\right), \\
& f_{3}=q^{3}-2 q^{4}+q^{5}-2 q^{7}+q^{9}+2 q^{11}+2 q^{13}-2 q^{15}+O\left(q^{17}\right) .
\end{aligned}
$$

Put $x=f_{1} / f_{3}$ and $y=f_{2} / f_{3}$. Then we can see that they satisfy no quadratic equations. In fact, by obtaining much more precise expressions for $f_{1}, f_{2}, f_{3}$, we can verify that $x$ and $y$ satisfy a quartic equation

$$
x^{3}-x^{2}\left(y^{2}+4\right)+2 x\left(y^{3}-2 y^{2}+2 y+3\right)-\left(y^{4}-4 y^{3}+8 y^{2}-8 y+7\right)=0,
$$

which is the defining equation of $X_{0}^{*}(144)$. Hence $X_{0}^{*}(144)$ is not hyperelliptic. We can give an alternative proof which uses Proposition 8. In the present case, we have

$$
G(T)=T^{8}-12 T^{7}+76 T^{6}-272 T^{5}+626 T^{4}-820 T^{3}+720 T^{2}-184 T+1
$$

and $w^{2}-G(z)=-864 q+\ldots$, hence again we conclude that $X_{0}^{*}(144)$ is not hyperelliptic.

Example 8. Let $N=207$. By Table 3 and Proposition 5 , we know that $X_{0}^{*}(207)$ is hyperelliptic. Let us compute the defining equation of $X_{0}^{*}(207)$. A basis of $S_{2}^{*}(207)$ is given by

$$
\begin{aligned}
f_{1}= & q+q^{2}-q^{3}-2 q^{4}-2 q^{5}-q^{6}-q^{7} \\
& -2 q^{8}-q^{9}-q^{10}+q^{12}-3 q^{13}+O\left(q^{14}\right),
\end{aligned}
$$




$$
\begin{aligned}
& f_{2}=q^{2}-2 q^{4}-q^{5}+q^{7}+q^{8}-q^{10}-2 q^{11}+O\left(q^{14}\right), \\
& f_{3}=q^{3}-q^{4}-2 q^{5}+q^{6}+q^{7}+q^{8}+q^{9}-q^{10}-4 q^{11}-q^{12}+3 q^{13}+O\left(q^{14}\right) .
\end{aligned}
$$

Then we compute $f_{2}^{2}-f_{1} f_{3}=O\left(q^{15}\right)$, or equivalently, $x^{2}-y=O\left(q^{9}\right)$ if we write $x=f_{2} / f_{3}$ and $y=f_{1} / f_{3}$. Since the degree of the divisors of poles of $x$ and $y$ are bounded by

$$
2 \cdot 3-2=4
$$

and since $x^{2}, y$ have a pole of order 2 at $\overline{i \infty}$, we see that the $f_{i}$ 's in fact satisfy a quadratic equation

$$
f_{2}^{2}-f_{1} f_{3}=0
$$

Hence again we find that $X_{0}^{*}(207)$ is hyperelliptic. Its defining equation is given by

$$
w^{2}=z^{8}-6 z^{7}+11 z^{6}-12 z^{5}+9 z^{4}-12 z^{3}+11 z^{2}-6 z+1,
$$

where we put

$$
z=\frac{f_{2}}{f_{3}} \quad \text { and } \quad w=\left(\frac{f_{3}}{q}\right)^{-1} \frac{d z}{d q}
$$

Using Proposition 8, we can show that $X_{0}^{*}(N)$ is not hyperelliptic for all $N$ in (8) (see Table 6 for data of Fourier coefficients). Hence we have

Theorem. Assume that $X_{0}^{*}(N)$ is of genus $\geq 3$. Then $X_{0}^{*}(N)$ is hyperelliptic if and only if $N=136,171,207,252,315,176$ or 279 . For $N \neq 207$ and $N \neq 315$, the hyperelliptic involution of $X_{0}^{*}(N)$ is of type $V$ in the notation of Section 2; namely, $V=S_{2} W_{8} S_{2}$ for $N=136, V=S_{2} W_{16} S_{2}$ for $N=176$, and $V=S_{3} W_{9} S_{3}^{2}$ for $N=171,252$ and 279. Their defining equations are given in Table 4 below.

Table 4

\begin{tabular}{ccc}
\hline$N$ & Defining equation $w^{2}=f(z)$ of $X_{0}^{*}(N)$ & $\begin{array}{c}\text { Discriminant of } \\
f(z)\end{array}$ \\
\hline 136 & $w^{2}=z(z+1)\left(z^{2}+3 z-2\right)\left(z^{4}+4 z^{3}+5 z^{2}+2 z-4\right)$ & $-2^{28} 17^{3}$ \\
171 & $w^{2}=\left(z^{2}-z+1\right)\left(z^{6}+z^{5}+2 z^{4}-7 z^{3}-2 z^{2}-3 z+9\right)$ & $2^{16} 3^{6} 19^{4}$ \\
207 & $w^{2}=z^{8}-6 z^{7}+11 z^{6}-12 z^{5}+9 z^{4}-12 z^{3}+11 z^{2}-6 z+1$ & $-2^{16} 3^{6} 23^{3}$ \\
252 & $w^{2}=\left(z^{2}+3\right)\left(z^{2}-z+1\right)\left(z^{4}-5 z^{3}+8 z^{2}-7 z+7\right)$ & $2^{28} 3^{4} 7^{4}$ \\
315 & $w^{2}=\left(z^{4}+z^{3}+3 z^{2}+z+1\right)\left(z^{4}+5 z^{3}+3 z^{2}+5 z+1\right)$ & $-2^{16} 3^{6} 5^{2} 7^{3}$ \\
176 & $w^{2}=z\left(z^{3}-4 z+4\right)\left(z^{3}-2 z^{2}+2\right)\left(z^{3}+2 z^{2}-2\right)$ & $-2^{40} 11^{5}$ \\
279 & $w^{2}=\left(z^{6}-z^{5}+z^{4}+2 z^{3}-z^{2}+1\right)$ & \\
& $\times\left(z^{6}+3 z^{5}+5 z^{4}+6 z^{3}+7 z^{2}+12 z+9\right)$ & $2^{24} 3^{8} 31^{6}$ \\
\hline
\end{tabular}

Remark 4. Let $N$ be an integer which is in Table 3. Then $X_{0}^{*}(N) /\langle V\rangle$ is of genus 2 for $N=207,315,160,216,630,336$ and 360 . We also give their defining equations in Table 5. 
Table 5

\begin{tabular}{lll}
\hline$N$ & Defining equation $w^{2}=f(z)$ of $X_{0}^{*}(N)$ & \multicolumn{1}{c}{$\begin{array}{c}\text { Discriminant of } \\
f(z)\end{array}$} \\
\hline 207 & $w^{2}=(z-1)(z+3)\left(z^{4}-2 z^{3}-5 z^{2}+6 z-3\right)$ & $-2^{12} 3^{6} 23^{3}$ \\
315 & $w^{2}=(z-3)(z+1)\left(z^{2}-z+1\right)\left(z^{2}+3 z-3\right)$ & $-2^{12} 3^{6} 5^{4} 7^{3}$ \\
160 & $w^{2}=\left(z^{2}+4\right)\left(z^{2}-2 z+2\right)\left(z^{2}+2 z+2\right)$ & $-2^{26} 5^{4}$ \\
216 & $w^{2}=\left(z^{2}-3 z+3\right)\left(z^{3}-3 z^{2}+3 z+3\right)$ & $2^{4} 3^{8}$ \\
630 & $w^{2}=\left(z^{2}+z-1\right)\left(z^{4}-z^{3}+2 z^{2}+7 z+7\right)$ & $2^{12} 3^{6} 5^{3} 7^{2}$ \\
336 & $w^{2}=\left(z^{2}-3\right)\left(z^{4}-11 z^{2}+32\right)$ & $2^{23} 3 \cdot 7^{2}$ \\
360 & $w^{2}=\left(z^{2}+3\right)\left(z^{2}-z+4\right)\left(z^{2}+z+4\right)$ & $-2^{18} 3^{3} 5^{2}$
\end{tabular}

Table 6

\begin{tabular}{rlr}
\hline$N$ & \multicolumn{1}{c}{ A basis of $S_{2}^{*}(N)$} & $d_{1}$ \\
\hline 162 & $(1,0,0,0,-4,-3,-4,-2,0,4,1,3)$ & 10 \\
& $(0,1,0,-1,-1,-3,0,-1,0,1,1,3)$ & \\
& $(0,0,1,0,-2,-1,-1,0,0,2,1,1)$ & -760 \\
196 & $(1,0,0,-1,-2,-2,-3,0,0,2,-1,0)$ & \\
& $(0,1,0,0,-2,-2,-3,1,1,2,1,0)$ & 49600 \\
& $(0,0,1,0,-2,-1,0,0,0,2,0,-1)$ & \\
240 & $(1,0,0,-2,-1,0,-1,0,-2,0,0,-2)$ & \\
& $(0,1,0,0,-2,-1,-2,0,0,3,-2,0)$ & \\
& $(0,0,1,-2,0,0,3,0,-3,0,0,-2)$ & \\
294 & $(1,0,0,0,-1,-1,-3,-1,-2,-2,-1,1)$ & \\
& $(0,1,0,0,-1,-1,0,-3,0,-3,0,0)$ & \\
& $(0,0,1,1,0,-1,-3,-3,-3,-1,1,0)$ & \\
420 & $(1,0,0,-1,0,-1,-1,0,0,-1,-2,0)$ & \\
& $(0,1,0,0,-2,-1,0,1,0,1,0,0)$ & \\
& $(0,0,1,0,-1,-1,0,0,-1,1,2,-1)$ & \\
476 & $(1,0,0,-1,-1,0,0,0,-1,-1,0,0)$ & \\
& $(0,1,0,0,-1,0,-1,1,-2,-1,-2,0)$ & \\
& $(0,0,1,0,0,-1,-1,0,0,0,-2,-1)$ & \\
\hline 630 & $(1,0,0,0,0,-1,0,-2,-2,1,-2,2,0,-1,-2,3,-5,1)$ & 24354 \\
& $(0,1,0,0,0,0,0,-3,0,-1,-2,0,2,-1,0,2,-4,0)$ & \\
& $(0,0,1,0,0,-1,-1,0,0,0,-1,1,-1,1,-1,0,-3,0)$ & \\
& $(0,0,0,1,0,0,1,-3,-1,0,-2,0,3,-1,0,4,-3,1)$ & \\
& $(0,0,0,0,1,-1,0,-1,-1,1,0,2,1,0,-2,4,-4,0)$ & \\
\hline & &
\end{tabular}

In Table 6 , we give a basis of $S_{2}^{*}(N)$ explicitly for all $N \neq 144$ in (8) (for $N=144$, see Example 7).

If $f_{i}(\tau) \in S_{2}^{*}(N)(1 \leq i \leq g)$ has the Fourier expansion $f_{i}(\tau)=$ $\sum_{n \geq 1} a_{n}^{(i)} q^{n}$, we give its Fourier coefficients by $\left(a_{1}^{(i)}, a_{2}^{(i)}, \ldots, a_{r}^{(i)}\right)$ with $r=$ $3 g+3$. Note that for all $N$ in Table 6 , the $f_{i}$ 's are of the form (6), and $r=3 g+3$ is the lowest bound to be able to calculate $d_{1}$ (see (9) and Proposition 8). We have $d_{1} \neq 0$ for all $N$ in (8). 
Acknowledgements. The author would like to express his thanks to Professors K. Hashimoto and F. Momose for their valuable suggestions and warm encouragement.

\section{References}

[1] A. O. L. Atkin and J. Lehner, Hecke operators on $\Gamma_{0}(m)$, Math. Ann. 185 (1970), $134-160$.

[2] A. O. L. Atkin and D. J. Tingley, Numerical tables on elliptic curves, in: Modular Functions of One Variable IV, B. Birch and W. Kuyk (eds.), Lecture Notes in Math. 476, Springer, Berlin, 1975, 74-144.

[3] P. Deligne et M. Rapoport, Les schémas de modules de courbes elliptiques, in: Modular Functions of One Variable II, P. Deligne and W. Kuyk (eds.), Lecture Notes in Math. 349, Springer, Berlin, 1973, 143-316.

[4] R. Fricke, Die Elliptischen Funktionen und ihre Anwendungen, Teubner, Leipzig and Berlin, 1916.

[5] Y. Hasegawa, Table of quotient curves of modular curves $X_{0}(N)$ with genus 2, Proc. Japan Acad. Ser. A 71 (1995), 235-239.

[6] - Modular abelian surfaces and hyperelliptic curves of genus two, preprint.

[7] Y. Hasegawa and K. Hashimoto, Hyperelliptic modular curves $X_{0}^{*}(N)$ with square-free levels, Acta Arith. 77 (1996), 179-193.

[8] H. Hijikata, Explicit formula of the traces of Hecke operators for $\Gamma_{0}(N)$, J. Math. Soc. Japan 26 (1974), 56-82.

[9] P. G. Kluit, Hecke operators on $\Gamma^{*}(N)$ and their traces, Dissertation of Vrije Universiteit, Amsterdam, 1979.

[10] J. Lehner and M. New man, Weierstrass points of $\Gamma_{0}(N)$, Ann. of Math. 79 (1964), $360-368$

[11] N. Murabayashi, On normal forms of modular curves of genus 2, Osaka J. Math. 29 (1992), 405-418.

[12] A. P. Ogg, Hyperelliptic modular curves, Bull. Soc. Math. France 102 (1974), 449462.

[13] M. Yamauchi, On the traces of Hecke operators for a normalizer of $\Gamma_{0}(N)$, J. Math. Kyoto Univ. 13 (1973), 403-411.

Department of Mathematics

Waseda University

3-4-1, Okubo Shinjuku-ku

Tokyo 169, Japan

E-mail: hase@mn.waseda.ac.jp

Received on 22.11.1996

and in revised form on 21.2.1997

(3082) 